Running Head: Culture and reactions to suicide ideation

\title{
Cultural Differences in Reactions to Suicide Ideation: A Mixed Methods Comparison of Korea and Australia
}

\author{
Soontae $\mathrm{An}^{1}$ \\ Tegan Cruwys*2,3 \\ Hannah Lee ${ }^{1}$ \\ Melissa Xue-Ling Chang ${ }^{3}$
}

1. Division of Communication and Media, College of Social Sciences, Ewha Womans University, Seoul, Korea

2. Research School of Psychology, The Australian National University, Canberra ACT 2601

3. School of Psychology, University of Queensland, Brisbane QLD 4072

* Corresponding author: Dr Tegan Cruwys, tegan.cruwys@anu.edu.au, Research School of Psychology, The Australian National University, Canberra ACT 2601, AUSTRALIA.

Citation: An, S., Cruwys, T., Chang, M. X-L., \& Lee, H. (in press, accepted 24 May 2019). Cultural differences in reactions to suicide ideation: A mixed methods comparison of Korea and Australia. Archives of Suicide Research.

This work was supported by the Ministry of Education of the Republic of Korea and the National Research Foundation of Korea (NRF-2018S1A5A2A01028437) as well as the Australian Research Council (DE160100592). 


\begin{abstract}
Objective: There is evidence for cultural differences in mental health symptoms and helpseeking, but no past research has explored cultural differences in how people react to suicide ideation communicated by others. Layperson reactions are critical, because the majority of people who experience suicide ideation disclose to friends or family.

Methods: Participants were 506 people aged 17-65 recruited from Australia and Korea who completed an experiment in which they responded to a friend who was experiencing either subclinical distress or suicidal ideation.

Results: Korean participants did not differentiate between the subclinical and suicidal targets, whereas Australian participants showed more concern for the suicidal target. For both targets, Korean participants were more likely to recommend passive coping strategies ("Time will solve everything" or "Cheer up") while Australian participants were more likely to recommend active coping strategies ("Let's talk" or "See a doctor").

Conclusion: This study provides the first evidence of cultural differences in the way people typically respond to disclosures of suicide ideation, and suggests that unhelpful and inappropriate recommendations are commonplace.
\end{abstract}

Keywords: help seeking; coping strategies; social support; mental health; depression 


\section{Cultural Differences in Reactions to Suicide Ideation: A Mixed Methods Comparison of Korea and Australia}

Suicide is a major public health concern, with approximately 800,000 people dying from suicide each year (World Health Organization, 2017). Studies have suggested that suicidal ideation is one of the strongest predictors of suicide attempts (Nock et al., 2008), and that a majority of people who attempt suicide communicate their intent to members of their social network (Hawton, Houston, \& Shepperd, 1999; Isometsa, 2001). For these reasons, investigating how laypeople respond when significant others communicate suicidal ideation presents a key opportunity for suicide prevention efforts. However, very few studies have examined how people communicate and respond to suicidal ideation, or patterns of helpseeking (Goldston et al., 2008). In addition, most of these studies have been conducted in Western cultural contexts. To the best of our knowledge, no studies have looked at crosscultural differences in how individuals respond to significant others' suicidal ideation. The present research fills this gap, examining differences between people from an East-Asian versus Western Caucasian cultural context in perceptions of significant others who communicate suicidal ideation, and the types of help provided in these situations. In examining these research questions, we focus on Korea in particular (an East-Asian country) because suicide rates in Korea have risen dramatically in recent years, with Korea having the highest suicide rate of all OECD nations (25.8 per 100,000; OECD, 2018). We contrast Korea to Australia, a Western and predominantly Caucasian country with a comprehensive national suicide prevention initiative (Matsubayashi \& Ueda, 2011) and a much lower suicide rate (12.8 per 100,000; OECD, 2018).

\section{How People Respond to the Disclosure of Suicidal Ideation}

In order to respond appropriately to a person expressing suicidal ideation, a person must first appraise the communication itself (e.g., as sufficiently serious to indicate a need for 
support) and what, if anything, they should do about it (e.g., whether the person should seek help and whom the person should seek help from; Chu, Hsieh, \& Tokars, 2011; Freedenthal \& Stiffman, 2007; Owens et al., 2011). The few studies that have investigated responses to communications of suicidal ideation found that the most common reactions were disbelief, denial, avoidance, or attempts to interact as if nothing has happened (Cowgell, 1977; Owens et al., 2011; Rudestam, 1971). More specifically, Owens et al. (2011) found that when responding to suicidal crisis, individuals typically go to great lengths to avoid pathologizing the distress of significant others and push back the threshold for help-seeking (i.e., suggesting people seek help only when the symptoms become unmanageable). Other studies have also suggested that displays of suicidal ideation are often met with interpersonal rejection (Lester \& Walker, 2006; Walker, Lester, \& Joe, 2006). Despite this, people who have experienced a suicidal crisis typically report that support from their friends and relatives is as helpful as professional support (Eagles, Carson, Begg \& Naji, 2003). Taken together, these studies suggest that lay responses to suicidal ideation can vary enormously in terms of how helpful they are to a person experiencing suicidality.

\section{Culture and Reactions to Suicidal Ideation}

Culture plays a role in the prevalence, etiology, and expression of suicidal thoughts (Colucci, 2013; Hjelmeland, 2010, 2011), as well as patterns of help-seeking for suicidal ideation (Chu et al., 2011; Goldston et al., 2008). That is, culture may influence what kinds of signs are interpreted as suicidal risk, and accordingly, the threshold of severity that triggers recognition of a need for help (Jorm, Ross, \& Colucci, 2018; Tzeng \& Lipson, 2004). Furthermore, culture may influence the types and sources of help that one perceives to be acceptable and the least stigmatizing (Freedenthal \& Stiffman, 2007). Although these findings suggest important cultural variation, the few cross-cultural studies that do exist have focused on what a person with suicidal ideation would hypothetically do (e.g., Chu et al., 
2011; Han, Batterham, Calear, and Ma, 2018) or cross-cultural examinations of first-aid guidelines using DELPHI methodology (e.g., Colucci, Kelly, Minas, Jorm, \& Chatterjee, 2010; Colucci, Kelly, Minas, Jorm, \& Nadera, 2010; Colucci, Kelly, Minas, Jorm, \& Suzuki, 2011). However, no research has examined cultural differences in how lay people respond to others' disclosure of suicidal ideation.

Nevertheless, there are some indications that relevant cultural differences exist in conceptualizations of suicide and recognition of warning signs. Specifically, East Asians are more likely to normalize, deny, and tolerate suicidality (Colucci \& Lester, 2013; Chu et al., 2011; Tzeng \& Lipson, 2004). For example, Chu et al. (2011) found that compared to Latino Americans, Asian Americans with suicidal ideation or attempts tended to underestimate the severity of these warning signs and were less likely to seek and perceive a need for help. Indeed, Asian Americans have been termed "hidden ideators" who, relative to Caucasian Americans, tend to conceal suicidal ideation and are less likely to disclose such thoughts to others (Morrison \& Downey, 2000). Furthermore, in East-Asian culture, suicide is often viewed as an immoral or weak behavior (An \& Lee, 2017a; Tzeng \& Lipson, 2004). These stigmatized cultural beliefs lead to negative attitudes and reactions toward suicidal people (Domino, 2005; Eskin, Voracek, Stieger, \& Altinyazar, 2011). This stigma may also be a barrier to help seeking, such that people in East-Asian cultures may be more likely to attempt to endure or overcome crisis situations (using passive help-seeking) rather than seek professional help (active help-seeking) (Linda, Marroquín \& Miranda, 2012). An and Lee (2017b) found that Koreans tend to view suicidal crisis as a problem not requiring professional support, but one to be tolerated and endured by oneself.

Overall, although these studies have not examined suicide ideation in significant others, the literature suggests that East-Asians compared to Westerners may be less likely to perceive suicidality as a serious problem and less likely to seek help. Accordingly, relative to 
Western Caucasians, we might expect East-Asians to be (a) less concerned about suicidal ideation communicated by significant others, (b) less likely to perceive that a person expressing suicidal ideation needs help, and thus (c) less likely to recommend help-seeking.

\section{The Current Study}

We conducted an experiment with Koreans and Australians to investigate the influence of culture on how people respond to a significant other who communicates suicidal ideation (versus subclinical distress). Given that social media use is related to well-being and health behaviour (Chou, et al., 2009; Dolev-Cohen \& Barak, 2013; Valkenburg, Peter, \& Schouten, 2006), and people under 40 have more social interaction via social media than face-to-face (Hall, 2018), we presented the communication from the significant other (called the target below) through a social media platform in the vignette. We expected that, compared to Australian participants, Korean participants would show less capacity to differentiate (H1) and effectively respond to (H2) significant others expressing suicidal ideation:

H1: Culture will interact with message type to predict level of concern, such that Australians, but not Koreans, will have elevated concern for a target expressing suicidal ideation (compared to a target expressing subclinical distress).

$\mathrm{H} 2$ : Culture will interact with message type to predict coping advice, such that Koreans, compared to Australians, will be less likely to recommend active help seeking, particularly to a target expressing suicidal ideation (compared to a target expressing subclinical distress).

\section{Method}

\section{Experimental Design and Vignette Development}

The study had a 2 (target presentation: subclinical distress vs. suicide ideation) $\times 2$ (culture: Australian vs. Korean) between-subjects design. The vignettes were designed in 
consultation with clinical psychologists (authors 2 and 4) such that the target described their experience in terms of distinctive mental health presentations: (1) subclinical symptoms related to an acute stressor, or (2) suicide ideation. All vignettes were based on the same setting (the breakdown of an intimate relationship), however the message sender in each vignette reacted differently, describing key indicators of their mental health status. To make the break-up situation gender-neutral, we used unisex names: the message sender, target, was named Alex in Australia and Ji-won in Korea.

The study used an instant messaging service as a media platform to present the target communicating distress. This is because among different types of social media platforms, instant messaging services are most appropriate to discuss private matters such as break-ups, rather than more open platforms such as Facebook (Quan-Haas \& Young, 2010). The interface was designed to mimic popular instant messaging services in each country, such as WhatsApp and Kakao Talk.

Apart from the key mental health symptoms described in instant messages, the structure and the length of vignettes were identical, with a length of approximately 38 words. All vignettes followed the same structure: the first sentence described the acute stressor; the second sentence revealed the target's emotional response; the third sentence further explained the problem; the fourth sentence described key symptoms; and the fifth sentence highlighted the level of psychological distress. Figure 1 displays the English version of the two vignettes in the instant message format.

$<$ Insert Figure 1 here $>$

\section{Participants}

We sought to recruit a diverse community sample of adults in both Korea and Australia. Australian participants were recruited using two strategies. First, a university participation pool was utilized, including both community members (who received a financial 
incentive) and undergraduate students (who received course credit as an incentive). Second, in order to improve the representativeness of the sample, a nation-wide market research panel was utilized to recruit Australians aged 25 and over who received a financial incentive. We limited Australian participants to Caucasians with Australian citizenship, because Australia has a multicultural population with a large proportion of first and second-generation residents from Asian countries. Korean data were gathered by a research firm with the largest nationwide representative panel in Korea. The firm utilized a panel of 75,000 participants and selected samples using random and quota sampling methods to represent the Korean population in terms of age and gender. Survey notification was sent via e-mail and SMS systems with a link to a survey URL and participants received a financial incentive.

A total of 506 adults $(\mathrm{N}=240$ Australians, $47.6 \% ; \mathrm{N}=264$ Koreans, 52.4\%) participated in the study. In general, Australian and Korean samples demonstrated similar characteristics, as shown in Table 1. Australian participants were $35.1 \%$ male and ranged in age from 17 to $65(M=29.21 ; S D=14.92)$. Approximately $10.9 \%$ of Australian participants $(N=26)$ reported that they had personally attempted suicide in the past, and $6.3 \%(N=15)$ reported severe current depression symptoms. Korean participants were $44.3 \%$ male and ranged in age from 18 to $64(M=34.35 ; S D=12.53)$. Approximately $10.2 \%$ of Korean participants $(N=27)$ reported that they had personally attempted suicide in the past, while $3.8 \%(N=10)$ reported severe current depression symptoms.

$<$ Insert Table 1 here $>$

\section{Procedure and Measures}

Participants completed the survey in English (Australian participants) or Korean (Korean participants). Back translation procedures were used when translating the English questionnaire to Korean. Back translation was conducted independently by two KoreanEnglish bilingual communication researchers (authors 1 and 3), and the questionnaire was 
revised until equivalence and relevance of all the questions were achieved.

All participants were randomly assigned to one of the two vignettes and were given the following instructions: "Imagine that you have received the following instant message from Alex [Ji-won], one of your close friends. Please read the following closely and answer the questions." After reading the vignette, participants were asked about their perceptions of the message sender. Specifically, the dependent variables were quantitative and qualitative measures of (1) how participants' perceived Alex's experience, and (2) what response participants thought was appropriate ${ }^{1}$.

Qualitative measures. Participants were first asked two open-ended questions about the target: "What state of mind is Alex in and what problems do you think Alex has?" and "Suppose Alex is someone close to you. What would you say to Alex?"

\section{Quantitative measures.}

Level of Concern. Participants' levels of concern for the message sender was measured using three items on a 5-point scale from 1 (not at all) to 5 (very much): 1) "If you were a close friend to Alex, how concerned would you be about Alex?"; 2) "How much unpleasant emotion do you think Alex would feel in this situation?" and 3) "Do you think unpleasant emotions would interfere with Alex's overall well-being?’. The level of concern was an average of these three items: Australian Cronbach's $\alpha=0.81(M=4.08, S D=0.71)$ and Korean Cronbach's $\alpha=0.80(M=3.88, S D=0.63)$.

Coping Advice. Participants were asked: "Imagine the first thing you said to Alex was: "I am so sorry to hear that you're having a tough time. If you were a close friend of Alex, what advice would you give next?" Participants were asked to select the top advice they would give from nine examples, based on which you would be most likely to

\footnotetext{
${ }^{1}$ There was another experimental condition (a clinical depression vignette) also included in the experiment. However, this was not focal to our hypotheses in the current study and is discussed elsewhere (Cruwys, An, Chang, \& Lee, 2018).
} 
say. Participants were asked to rank order nine examples of coping advice. Their first choice of response was then recoded into two categories, corresponding to passive coping: "Time will solve everything," "Keep yourself busy" and "Try hiking or some light exercise," or active coping: "Spend time with people close to you," "See your doctor," "Seek psychological counseling," "Seek prescription medicine," "Call the Suicide Helpline," and "Go to a hospital."

Depression symptoms. Participants' current mental health status was assessed using the Patient Health Questionnaire-9 (Kroenke, Spitzer, \& Williams, 2001). The nine items asked people to indicate the frequency of depressive symptoms (as outlined in the DSM-IV, APA, 2000) that a person had experienced in the preceding two weeks. Symptoms were rated on a 4-point scale from 0 "Not at all" to 3 "Nearly every day": Australian Cronbach's $\alpha=$ $0.89(M=8.31, S D=6.40)$ and Korean Cronbach's $\alpha=0.91(M=7.85, S D=6.32)$

Suicide contact. Participants were asked three questions to assess their degree of personal contact with suicide, each with response options of "Yes" and "No". They were "Have you ever attempted suicide?", "Has anyone close to you (e.g., family member or friend) ever attempted suicide?", and "Has anyone close to you (e.g., family member or friend) ever died from suicide?" A suicide contact variable was coded as follows: (1) no contact with suicide, (2) close friend or family attempted suicide or died by suicide, (3) personal past suicide attempt (Holmes, Corrigan, Williams, Canar \& Kubiak, 1999).

\section{Statistical Analyses}

Quantitative analysis. First, to determine whether culture interacted with vignette type to predict level of concern (H1), we conducted a two-way analysis of covariance (ANCOVA). Age, gender, education, income, depression level, and suicide contact were entered as covariates. To explore whether culture interacted with vignette type to predict coping advice (H2), a hierarchical logistic regression was utilized to predict coping advice, 
with age, gender, education, income, depression level, and suicide contact as covariates. As reported below, both of these analyses were repeated with and without covariates to assess their robustness. All the interaction terms were entered with mean-centered values.

Qualitative analysis. To shed further light on participants' reactions to the target, we investigated participants' qualitative responses by conducting a semantic network analysis. Semantic network analysis is a type of visual text analysis that determines the most frequently used words from unstructured text data and represents the textual data as a network of objects (van Atteveldt, 2008). Compared with textual representation, the visual analytic approach is not only superior in terms of a fast and better understanding of semantic structures (Drieger, 2013), but also helps to reveal intrinsic meanings shared by members of a social group (Leydesdorff \& Welbers, 2011).

First, we extracted morpheme words (three parts of speech: nouns, verbs, and adjectives) from unstructured data (open-ended responses) using TEXTOM software that can analyze textual data in both English and Korean. During this step, we eliminated all noninformative words (e.g. if, and, that, a/an, the, to, is) and converted synonyms into specific unified terms. For example, the words "disbelief," "distrust," and "doubt" were all represented by "distrust." Sentences that conveyed similar meanings were also coded as one representative sentence. For example, "meet someone," "find someone else," and "find the right person for you" were all coded as "find someone new." Furthermore, we capitalized the first letters to link phrasal verbs that consisted of more than one-word (e.g., "take your mind off" $\rightarrow$ "MindOff," "will get better" $\rightarrow$ "WillGetBetter").

We then conducted semantic network analysis to analyze qualitative responses in depth. To gain a better understanding of the association between a target's state of mind and the corresponding coping advice found in the participants' responses, the answers to the two open-ended questions were combined into one cell. The TEXTOM software creates a words- 
by-words matrix (a symmetrical co-occurrence matrix; one-mode) that yields the most frequently co-occurring words in the text answers. The one-mode matrix was then visualized by UCINET's NetDraw package in the form of a network map (Borgatti, Everett, \& Johnson, 2013).

In a semantic network analysis, the role of keywords in the network structure and the connection strength of the keywords are determined on the basis of the index of centrality (e.g., degree, closeness, and eigenvector). Degree centrality refers to the number of links to and from a node (Borgatti et al., 2013). A high-degree word is located at the center of the network (highly visible) and tends to be seen as an important keyword. The node size also indicates a word's frequency of occurrence, with bigger nodes representing terms that appear more frequently than others. Closeness centrality is the geodesic distance from one node to another and indicates how closely linked two nodes are (Freeman, 2000). A word with highcloseness is centrally located and close to many other words. In addition, the thicker the line connecting the words is, the more often a pair of words co-occurs in the text. Eigenvector centrality measures the overall influence of a node in the network; it assigns relative scores to all the nodes in a network such that greater weight is placed on links to more central words (Borgatti et al., 2013).

\section{Results}

\section{Concern for the Target}

The analyses for $\mathrm{H} 1$ are presented in Table 2. First, we checked the assumption of homogeneity of variances; the Levene's test showed that the residual variation was the same across the four groups, $F(3,497)=2.15, p=0.093$. Of the covariates, gender $F(1,497)=$ $16.04, p<0.001, \eta^{2}=0.03$, and contact experience $F(1,497)=10.24, p=0.001, \eta^{2}=0.02$, significantly predicted level of concern. Female participants and those with the more suicide contact had higher levels of concern. 
Step 2 revealed a significant main effect for culture $F(1,495)=6.30, p=0.012, \eta^{2}=$ 0.01 . Australian participants $(M=4.08, S D=0.71)$ reported significantly higher levels of concern for the targets than Korean participants $(M=3.88, S D=0.63)$. There was also a significant main effect of target presentation $F(1,495)=5.10, p=0.024, \eta^{2}=0.01$. Specifically, participants who read the message from the target with suicide ideation $(M=$ 4.03, $S D=0.64)$ expressed a higher level of concern than participants who read the subclinical distress message $(M=3.91, S D=0.70)$.

$<$ Insert Table 2 here $>$

Most relevant to $\mathrm{H} 1$ was the interaction between culture and target presentation $F(1,494)=8.15, p=0.004, \eta^{2}=0.02$. As shown in Figure 2, Australians reported significantly higher levels of concern for the target with suicide ideation $(M=4.22, S D=$ $0.66)$, than the subclinical distress target $(M=3.94, S D=0.73), t(239)=3.38, p=0.001$. Conversely, Koreans showed comparable levels of concern regardless of whether the target expressed suicide ideation $(M=3.86, S D=0.57)$ or subclinical distress $(M=3.89, S D=$ $0.69), t(263)=-0.43, p=0.668$. The pattern of results was the same without covariates: culture $F(1,501)=11.04, p=0.001, \eta^{2}=0.02$, target presentation $F(1,501)=5.23, p=0.023$, $\eta^{2}=0.01$, and the interaction between culture and target presentation $F(1,499)=8.13, p=$ $0.005, \eta^{2}=0.02$, were all statistically significant.

$<$ Insert Figure 2 here $>$

\section{Coping Advice for the Target}

The analyses for H2 are shown in Table 3. Control variables (age, gender, education, income, depression, and contact experience) were entered in $\operatorname{Model} 1\left(\chi^{2}(6)=38.89, p<.001\right.$, Nagelkerke $\left.R^{2}=0.10\right)$. Among the control variables, only contact experience was statistically significant $(\mathrm{B}=1.18, p=0.035$, OR: 1.18). Participants with more suicide contact were more likely to recommend active coping. Model 2 added culture and target presentation $\left(\Delta \chi^{2}(2)=\right.$ 
$32.73, p<.001$, Nagelkerke $R^{2}=0.18$ ). Culture was significantly associated with type of coping advice $(\mathrm{B}=-1.37, p<0.001$, OR: 0.25$)$. That is, Australian participants tended to recommend active coping, while Korean participants tended to recommend passive coping. The main effect of target presentation was not significant $(\mathrm{B}=0.32, p=0.098, \mathrm{OR}: 1.38)$. The final Model 3 added the interaction terms between culture and target presentation $\left(\Delta \chi^{2}(1)=0.87, p=0.352\right.$, Nagelkerke $\left.R^{2}=0.18\right)$. The interaction term was not a significant predictor $(\mathrm{B}=-0.36, p=$ 0.353, OR: 0.70). This is indicates that the main effect whereby Australians were more likely to suggest active coping and Koreans were more likely to suggest passive coping was not moderated by target presentation.

The pattern of results was the same without covariates: the culture was significantly associated with the likelihood of coping advice $(\mathrm{B}=-1.42, p<0.001$, OR: 0.24$)$, while target presentation $(\mathrm{B}=0.28, p=0.146$, OR: 1.32$)$ and the interaction between culture and target presentation ( $\mathrm{B}=-0.23, p=0.542$, OR: 0.79$)$ were not statistically significant.

$<$ Insert Table 3 here $>$

\section{Semantic Network Analysis of Open-ended Responses}

Figure 3 and Figure 4 show the frequency of Koreans' responses to the targets. "Breakup" $(\mathrm{EC}=0.59)$ was the most central word in Korean participants' reactions to the targets with the subclinical distress message. Similarly, for reactions to targets with suicide ideation, "Breakup" $(\mathrm{EC}=0.56)$ was also the most central word. This indicates that Korean participants were more concerned with responding to the context than to the warning signs of suicide crisis.

$<$ Insert Figures 3 and 4 here $>$

Unlike Koreans, the reactions of Australians differed according to the types of messages received. Australian participants' reactions to targets with subclinical distress, the 
most central word was "Breakup" $(\mathrm{EC}=0.36)$. However, "Depression" $(\mathrm{EC}=0.48)$ was the most central word in reactions to the targets with suicide ideation. Figures 5 and 6 display the frequency of responses by Australian participants.

\section{$<$ Insert Figures 5 and 6 here $>$}

Figures 7 and 8 illustrate the two contrasting approaches of Koreans and Australians in responding to the targets. As shown in Figure 7, the central word "Breakup" is linked to other nodes: "Find someone new" $(\mathrm{EC}=0.39)$ has the closest relationship with "Breakup", followed by "Cheer up" (EC = 0.26), "Forget" $(\mathrm{EC}=0.23)$, and "Time will solve" $(\mathrm{EC}=$ 0.22). Thus, most common response was to reflect the contextual event and recommend passive coping strategies. "Depression" $(\mathrm{EC}=0.23)$ and "Distressed" $(\mathrm{EC}=0.21)$ also have a close relationship to "Breakup", but active coping terms were not linked to these.

\section{$<$ Insert Figure 7 here $>$}

However, Australians' responses to the two situations show significant differences. As shown in Figure 8, for the subclinical distress, the central word "Breakup" is closely linked to "Let's talk" $(\mathrm{EC}=0.26)$, “Concerned" $(\mathrm{EC}=0.23)$, "I'm here for you" $(\mathrm{EC}=0.23)$ and "Taylor (ex) is worthless" (EC $=0.22)$. By contrast, for the suicidal target, the central word "Depression" co-occurs with such terms as "Let's talk" (EC = 0.32), "I'm here for you" (EC $=0.24)$, "Concerned" $(\mathrm{EC}=0.23)$, "You are a valuable person" $(\mathrm{EC}=0.20)$, "Will get better" $(\mathrm{EC}=0.20)$, and "Let's meet" $(\mathrm{EC}=0.18)$. Notably, "Suicidal" appears less frequently than "Depression," as shown by the node size, but both words are closely connected to "Need help" $(\mathrm{EC}=0.17)$. Also, it is noteworthy that Australian were more likely to respond to the target with co-occurring terms such as "Suicidal," "Beyond blue," (an Australian mental health service that offers crisis support) and "Seek doctor," whereas Korean participants did not. 
$<$ Insert Figure 8 here $>$

Taken together, the results of the semantic network analyses confirm the results of the quantitative analyses. Koreans were less likely to differentiate subclinical symptoms from suicide ideation, whereas Australians demonstrated elevated levels of concern for individuals expressing suicide ideation. Furthermore, Australians more frequently mentioned "Need help" coping advice for those with suicide ideation and offered active solutions such as "Let's talk" and "Beyond blue" when they identified signs of depression or suicidal thoughts. However, most Koreans offered passive coping advice such as "Time will solve" and "Forget", regardless of the situation. Korean participants were not only less likely to detect the warning signs of suicide, but also more likely to consider suicide crisis as a problem that can be overcome by the affected individuals themselves.

\section{Discussion}

This study was the first to investigate cultural differences in how laypeople react to the communication of suicide ideation. Consistent with H1, results revealed that Australians were more likely to differentiate between the expression of subclinical distress and suicide ideation, showing a greater level of concern for suicidal targets and being more likely to identify warning signs such as "Depression", "Suicidal”, "Struggling" and "Self Harm”. By contrast, Koreans reacted to targets with subclinical distress and suicide ideation in comparable ways, showing similar levels of concern for each, Koreans were more likely to describe either target's experience in terms of their social context, with the phrase "Break up" being most common in both conditions. A minority of Korean, but not Australian, participants also described the target in stigmatizing terms such as "Weak", "Pathetic" and "Complaining". The finding that Korean participants were insensitive to the difference between the target presentations and rarely identified warning signs for suicide warrants closer examination. 
Coping advice was also significantly different between the two countries. However, this was a main effect of culture, rather than an interaction as predicted in H2. Across both targets, Australians were more likely to suggest active coping strategies involving social and professional support. This finding was apparent both from quantitative analyses and the qualitative responses, where Australians were more likely to suggest "Let's talk", "I'm here for you" or "See a Doctor". By contrast, Koreans were more likely to suggest passive coping strategies such as waiting, keeping yourself busy, or self-help such as exercise. In the qualitative responses, "Time will solve everything", "Cheer Up" and "Forget" were the most frequent coping advice among Korean participants. Indeed, even for the suicidal target, most Koreans advised no specific help seeking.

It is also interesting to note that the response "Let's drink" was closely connected to "Breakup" among Korean participants. Although the node size of "Let's drink" was small because of its low frequency, the close distance and thicker line between the two words indicated a strong co-occurence. Considering the correlation between suicide attempts and alcohol use (Hufford, 2001), this association between "Breakup" and "Let's drink" is cause for concern. This speaks to a lack of awareness of the potential risk posed by alcohol for the suicidal target among these Korean participants. Such initial responses by significant others may be critical in setting the tone and direction in which suicidal people seek alleviation to their situation (Colucci et al., 2010; Jorm et al., 2018).

These results are consistent with a growing body of work documenting cultural differences in beliefs about negative emotion more generally (e.g., Chang et al., 2017; De Vaus et al., 2018). In collectivist East-Asian societies, accepting and overcoming negative emotions and hardships is often regarded as necessary for self-improvement and growth (Heppner et al., 2006). Accordingly, people in East-Asian cultures may be more likely to minimize, tolerate, or suppress negative emotion (Markus \& Kitayama, 1991; Matsumoto, 
Yoo, Nakagawa, 2008). This may have implications for suicide risk. For instance, one study found that Koreans with high levels of emotional suppression were more likely to have negative attitudes toward psychologist/psychiatric counseling for suicide prevention (An \& Lee, 2017b).

However, such views are not widely accepted in Western Caucasian societies, where experiences of positive emotions are perceived as the driving force behind personal growth (Diener \& Diener, 1996). In Western Caucasian societies, negative emotions and hardships are to be avoided (Bastian et al., 2012). These different cultural orientations towards negative emotions and hardships are likely to have an impact on how people from each culture understand warning signs for suicide and their preferred means to cope with suicide risk. This is in keeping with our finding that Koreans were less likely to distinguish between putatively healthy negative emotional experiences and warning signs for suicide.

Finally, these findings shed new light on the high rate of completed suicide in Korea. Specifically, we find that in Korea the typical reaction to the disclosure of suicide ideation is more likely to be inappropriate and unhelpful, encouraging people to cope without professional or social support. By contrast, in Australia - a country which has a national suicide prevention strategy and where mental health literacy initiatives have been widespread (World Health Organization, 2010) - typical reactions are more cognizant of the severity of suicide ideation and tended to encourage active coping. Future research seeking to address suicide risk in Korea may benefit from adapting and trialing suicide literacy programs in Korea that have shown promising outcomes in Australia (Jorm et al., 2003; Taylor-Rodgers \& Batterham, 2014). However, it will be important that such programs are modified in order to be culturally appropriate for the East-Asian context, including a consideration of cultural beliefs and norms about suicide and negative emotion.

Like all research, this study had a number of limitations. First, the findings can only 
speak to the specific cultural contexts examined (Korean and Australia), and should not be generalized as representative of all Eastern and Western cultural contexts. Second, although Australians were better than Koreans at differentiating signs of suicide and provided more appropriate coping advice, this difference should be interpreted cautiously. We did not investigate specific vulnerable groups - for instance, in Australia rates of suicide among youth and Indigenous Australians remains high, and this study did not specifically investigate these contexts. Further research is needed in order to evaluate how responses to suicide ideation in these groups might differ.

\section{Conclusions}

Suicide is a multifactorial phenomenon with social and cultural determinants (Hjelmeland \& Knizek, 2011). The current study highlights differences between Koreans and Australians in the understanding of, and capacity to respond effectively to, warning signs of suicide in one's social networks. People experiencing suicidal ideation are often encouraged to reach out to their friends and family, however, the reactions of those loved ones are all too often inadequate and unhelpful. This study speaks to the need for a new approach to interventions to address the high rates of completed suicide in Korea - by providing training and information so that people can provide appropriate support to their loved ones who are at risk of suicide. 
Vignette \# 1

Subclinical distress

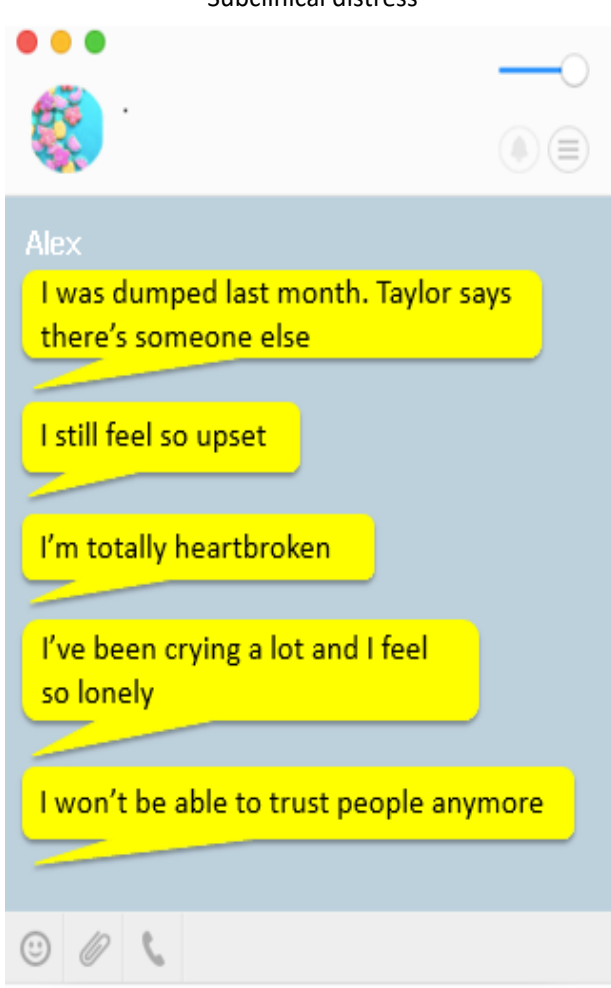

Vignette \# 2

Suicide ideation

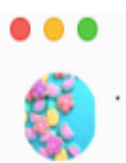

Alex

I was dumped last month. Taylor says there's someone else

I feel so numb. Like nothing matters

I'm totally heartbroken

I don't feel like eating and I can't sleep at night

I feel worthless and I don't think I can do this anymore

(:) 16

Figure 1. Experimental Materials 
Table 1. Sample Characteristics $(\mathrm{N}=506)$

\begin{tabular}{|c|c|c|c|}
\hline & Australian & Korean & Total \\
\hline \multicolumn{4}{|l|}{ Gender } \\
\hline Male & $85(35.1 \%)$ & $117(44.3 \%)$ & $202(39.9 \%)$ \\
\hline Female & $157(64.9 \%)$ & $147(55.7 \%)$ & $304(60.1 \%)$ \\
\hline \multicolumn{4}{|l|}{ Education Level } \\
\hline Year 10 or less & $15(6.2 \%)$ & $7(2.7 \%)$ & $22(4.3 \%)$ \\
\hline High School Certificate, including college student & $143(59.1 \%)$ & $37(14.0 \%)$ & $180(35.6 \%)$ \\
\hline Certificate or Diploma & $71(29.3 \%)$ & $58(22.0 \%)$ & $129(25.5 \%)$ \\
\hline Bachelor Degree & $7(2.9 \%)$ & $146(55.3 \%)$ & $153(30.2 \%)$ \\
\hline Postgraduate Degree & $6(2.5 \%)$ & $16(6.1 \%)$ & $22(4.3 \%)$ \\
\hline \multicolumn{4}{|l|}{ Income (AUD) } \\
\hline Less than $\$ 25,000$ & $56(23.4 \%)$ & $112(42.4 \%)$ & $168(33.4 \%)$ \\
\hline$\$ 25,000$ to $\$ 34,999$ & $11(4.6 \%)$ & $41(15.5 \%)$ & $52(10.3 \%)$ \\
\hline$\$ 35,000$ to $\$ 49,999$ & $26(10.9 \%)$ & $53(20.1 \%)$ & $79(15.7 \%)$ \\
\hline$\$ 50,000$ to $\$ 74,999$ & $34(14.2 \%)$ & $25(9.5 \%)$ & $59(11.7 \%)$ \\
\hline$\$ 75,000$ to $\$ 99,999$ & $34(14.2 \%)$ & $19(7.2 \%)$ & $53(10.5 \%)$ \\
\hline$\$ 100,000$ to 149,999 & $34(14.2 \%)$ & $9(3.4 \%)$ & $43(8.5 \%)$ \\
\hline$\$ 150,000$ or more & $44(18.4 \%)$ & $5(1.9 \%)$ & $49(9.7 \%)$ \\
\hline \multicolumn{4}{|l|}{ Reported Past Suicide Attempt } \\
\hline Yes & $26(10.9 \%)$ & $27(10.2 \%)$ & $53(10.5 \%)$ \\
\hline No & $213(89.1 \%)$ & $237(89.8 \%)$ & $450(89.5 \%)$ \\
\hline \multicolumn{4}{|l|}{ Know Someone who has Completed Suicide } \\
\hline Yes & $60(24.8 \%)$ & $46(17.4 \%)$ & $106(20.9 \%)$ \\
\hline No & $182(75.2 \%)$ & $218(82.6 \%)$ & $400(79.1 \%)$ \\
\hline \multicolumn{4}{|l|}{ Know Someone who has Attempted Suicide } \\
\hline Yes & $90(37.2 \%)$ & $57(15.9 \%)$ & $132(26.1 \%)$ \\
\hline No & $152(62.8 \%)$ & $335(84.1 \%)$ & $374(73.9 \%)$ \\
\hline \multicolumn{4}{|l|}{ Level of Depression Severity (PHQ-9 Score) } \\
\hline Minimal (0-4) & $81(33.8 \%)$ & $110(41.7 \%)$ & $191(37.9 \%)$ \\
\hline Mild (5-9) & $68(28.3 \%)$ & $63(23.9 \%)$ & $131(26.0 \%)$ \\
\hline Moderate (10-14) & $52(21.7 \%)$ & $53(20.1 \%)$ & $105(20.8 \%)$ \\
\hline Moderately severe (15-19) & $24(10.0 \%)$ & $28(10.6 \%)$ & $52(10.3 \%)$ \\
\hline Severe $(20-27)$ & $15(6.3 \%)$ & $10(3.8 \%)$ & $25(5.0 \%)$ \\
\hline
\end{tabular}


Table 2. ANCOVA Results on Level of Concern

\begin{tabular}{|c|c|c|c|c|c|}
\hline Variables & $d f$ & MS & $F$ & $p$ & $\eta_{p}^{2}$ \\
\hline \multicolumn{6}{|l|}{ Controls } \\
\hline Age & 1 & .80 & 1.91 & .168 & .00 \\
\hline Gender & 1 & 6.70 & 16.4 & .000 & .03 \\
\hline Education Level & 1 & .64 & 1.53 & .216 & .00 \\
\hline Income & 1 & .00 & .01 & .934 & .00 \\
\hline Depression Level & 1 & .29 & .68 & .410 & .00 \\
\hline Contact Experience & 1 & 4.28 & 10.24 & .001 & .02 \\
\hline \multicolumn{6}{|l|}{ Main Effects } \\
\hline Culture $(0=$ Australia, $1=$ Korea $)$ & 1 & 2.63 & 6.30 & .012 & .01 \\
\hline $\begin{array}{l}\text { Target presentation } \\
(0=\text { subclinical distress, } 1=\text { suicidal ideation })\end{array}$ & 1 & 2.13 & 5.10 & .024 & .01 \\
\hline \multicolumn{6}{|l|}{ Interaction } \\
\hline Culture $\mathrm{x}$ Target presentation & 1 & 3.40 & 8.15 & .004 & .02 \\
\hline
\end{tabular}

Note. $N=501$ (missing=5). 
$\longrightarrow$ Australian $\multimap-$ Korean

4.3

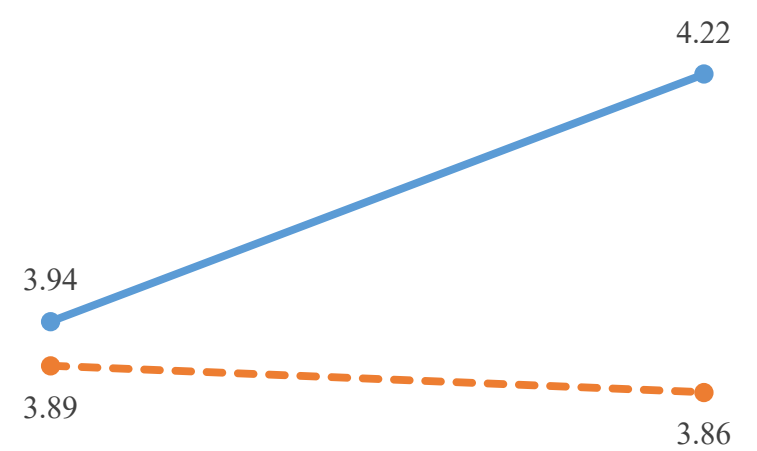

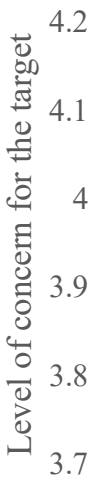

3.6

Subclinical distress

Suicidal ideation

Target presentation

Figure 2. Interaction between Culture and Target Presentation 
Table 3. Logistic Regression on Type of Coping Advice

\begin{tabular}{|c|c|c|c|c|c|c|}
\hline \multirow{2}{*}{$\begin{array}{l}\text { DV: Type of coping advice } \\
(0=\text { passive, } 1=\text { active })\end{array}$} & \multicolumn{2}{|c|}{ Model 1} & \multicolumn{2}{|c|}{ Model 2} & \multicolumn{2}{|c|}{ Model 3} \\
\hline & B & $\mathrm{OR}[\mathrm{CI}]$ & $\mathrm{B}$ & $\mathrm{OR}[\mathrm{CI}]$ & $\mathrm{B}$ & $\mathrm{OR}[\mathrm{CI}]$ \\
\hline \multicolumn{7}{|l|}{ Controls } \\
\hline Age $(0=$ male, $1=$ female $)$ & .00 & $1.00[.99$ & .00 & $1.00[.99$ & .00 & $1.00[.99$ \\
\hline Gender & -.06 & $1.02]$ & -.11 & $1.01]$ & -.11 & $1.02]$ \\
\hline Education & -.28 & $.94[.64$, & -.05 & $.90[.60$ & -.05 & $.90[.60$, \\
\hline Household Income & .11 & 1.39] & -.01 & $1.34]$ & -.01 & 1.34] \\
\hline Depression level & .10 & $.75^{* *}$ & .09 & $.95[.80$ & .10 & $.95[.80$, \\
\hline \multirow[t]{7}{*}{ Contact Experience } & .18 & {$[.66, .87]$} & .17 & $1.12]$ & .17 & $1.12]$ \\
\hline & & $1.11 *[1.01$ & & $.99[.89$ & & $.99[.89$, \\
\hline & & $1.22]$ & & 1.10] & & 1.09] \\
\hline & & $1.11[.94$ & & $1.09[.92$, & & $1.10[.93$ \\
\hline & & $1.30]$ & & $1.29]$ & & $1.30]$ \\
\hline & & $1.20 *[1.03$, & & $1.18 *[1.01$, & & $1.18^{*}[1.01$, \\
\hline & & $1.40]$ & & $1.38]$ & & $1.38]$ \\
\hline \multicolumn{7}{|l|}{ Main Effects } \\
\hline Culture & & & -1.36 & $.26^{* *}$ & -1.37 & $.25 * *$ \\
\hline$(0=$ Australia, $1=$ Korea $)$ & & & .31 & {$[.16, .42]$} & .32 & {$[.16, .42]$} \\
\hline Target presentation & & & & $1.36[.93$ & & $1.38[.94$ \\
\hline ( $0=$ subclinical distress, & & & & 1.99] & & 2.02] \\
\hline \multicolumn{7}{|l|}{$1=$ suicidal ideation) } \\
\hline \multicolumn{7}{|l|}{ Interaction } \\
\hline \multirow{2}{*}{$\begin{array}{c}\text { Culture } \\
\text { presentation }\end{array}$} & & & & & -.36 & $.70[.32$ \\
\hline & & & & & & $1.50]$ \\
\hline$-2 \mathrm{LL}$ & \multicolumn{2}{|c|}{654.59} & \multicolumn{2}{|c|}{621.86} & \multicolumn{2}{|c|}{620.99} \\
\hline Nagelkerke $\boldsymbol{R}^{2}$ & \multicolumn{2}{|c|}{.10} & \multicolumn{2}{|c|}{.18} & \multicolumn{2}{|c|}{.18} \\
\hline Model $\chi^{2}$ & \multicolumn{2}{|c|}{$38.89 * *(d f=6)$} & \multicolumn{2}{|c|}{$71.62 * *(d f=8)$} & \multicolumn{2}{|c|}{$72.49 * *(d f=9)$} \\
\hline$\Delta \chi^{2}$ & & $*(d f=6)$ & & $(d f=2)$ & & $(d f=1)$ \\
\hline
\end{tabular}

Note. $N=501$ (missing=5).

$* p<.05, * * p<.01$ 


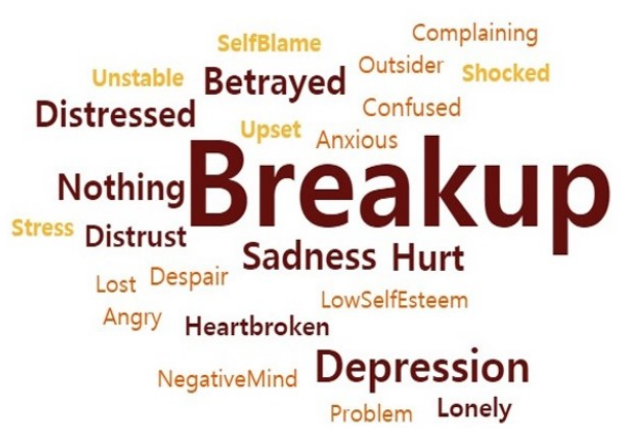

Subclinical distress

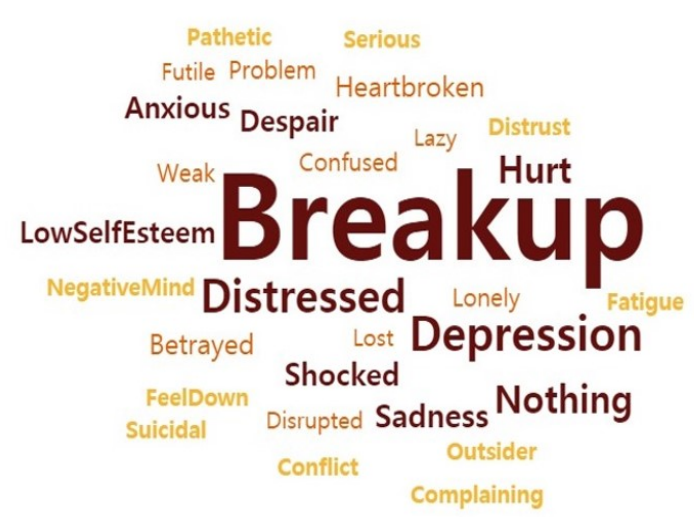

Suicidal ideation

Figure 3. Target's state of mind by Korean participants
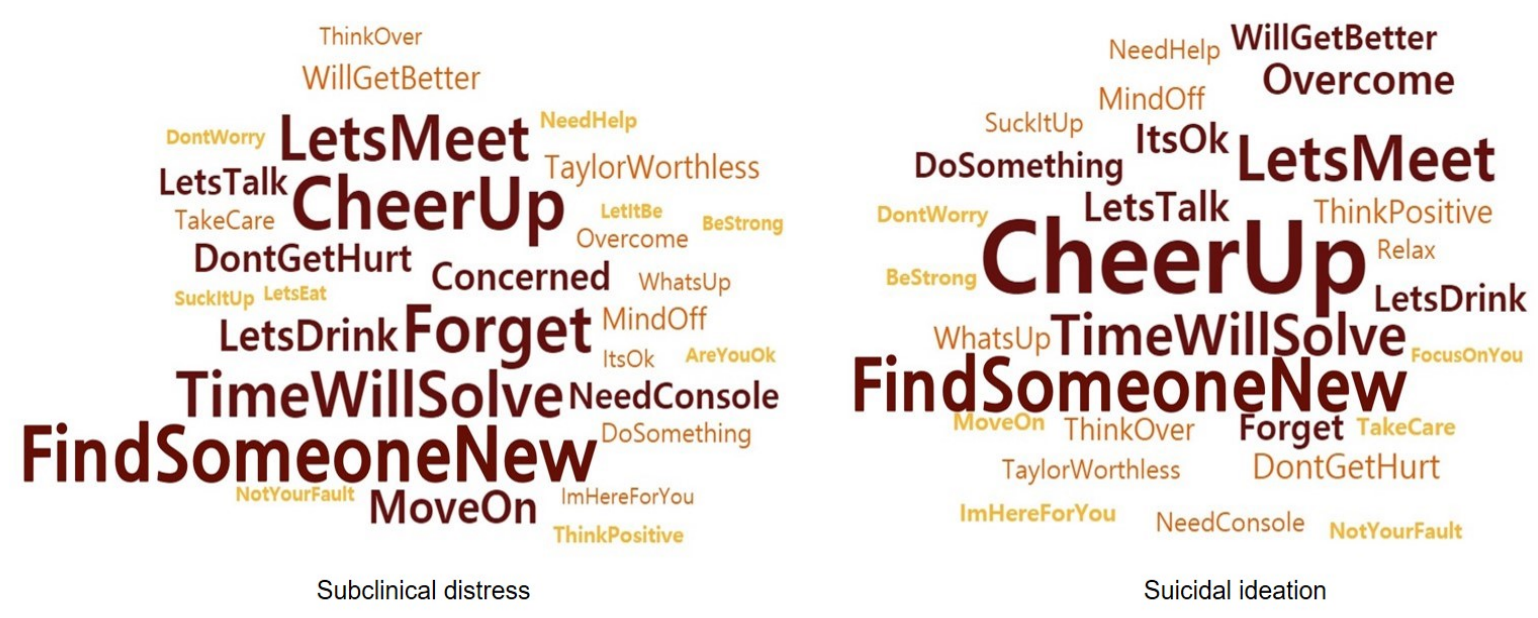

Figure 4. Coping advice by Korean participants

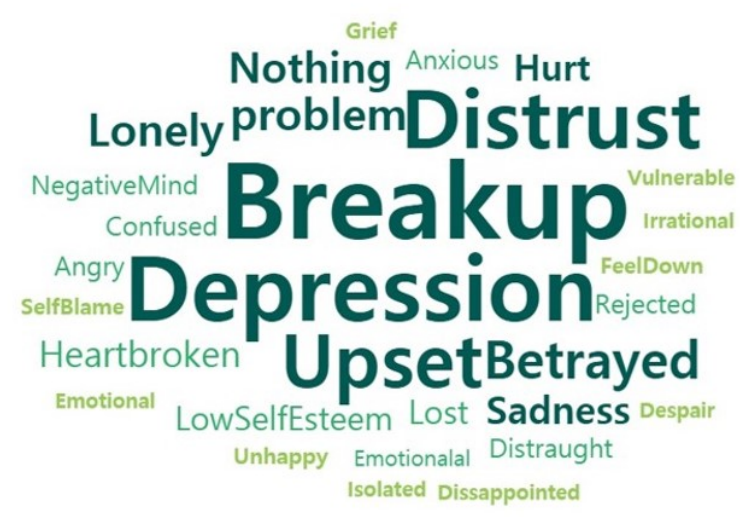

Subclinical distress

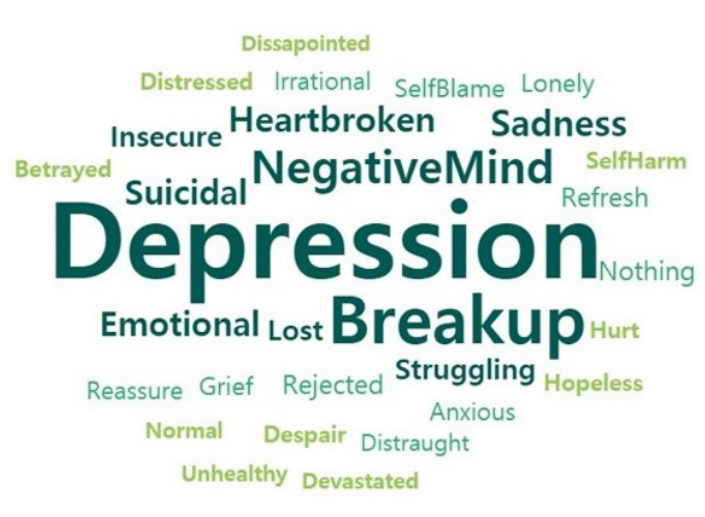

Suicidal ideation

Figure 5. Target's state of mind by Australian participants 


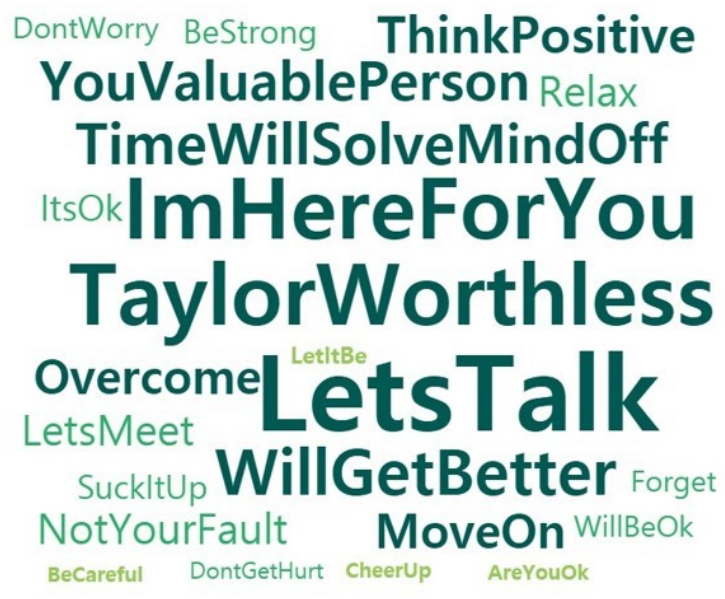

Subclinical distress

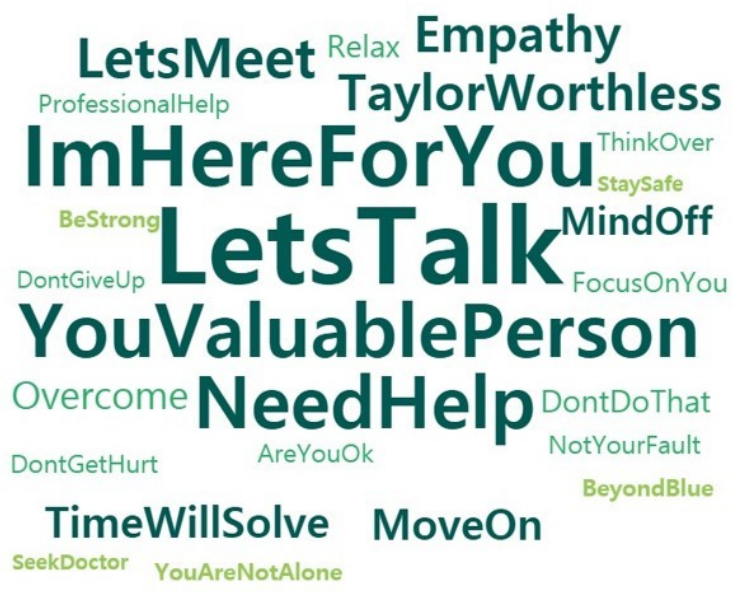

Suicidal ideation

Figure 6. Coping advice by Australian participants 


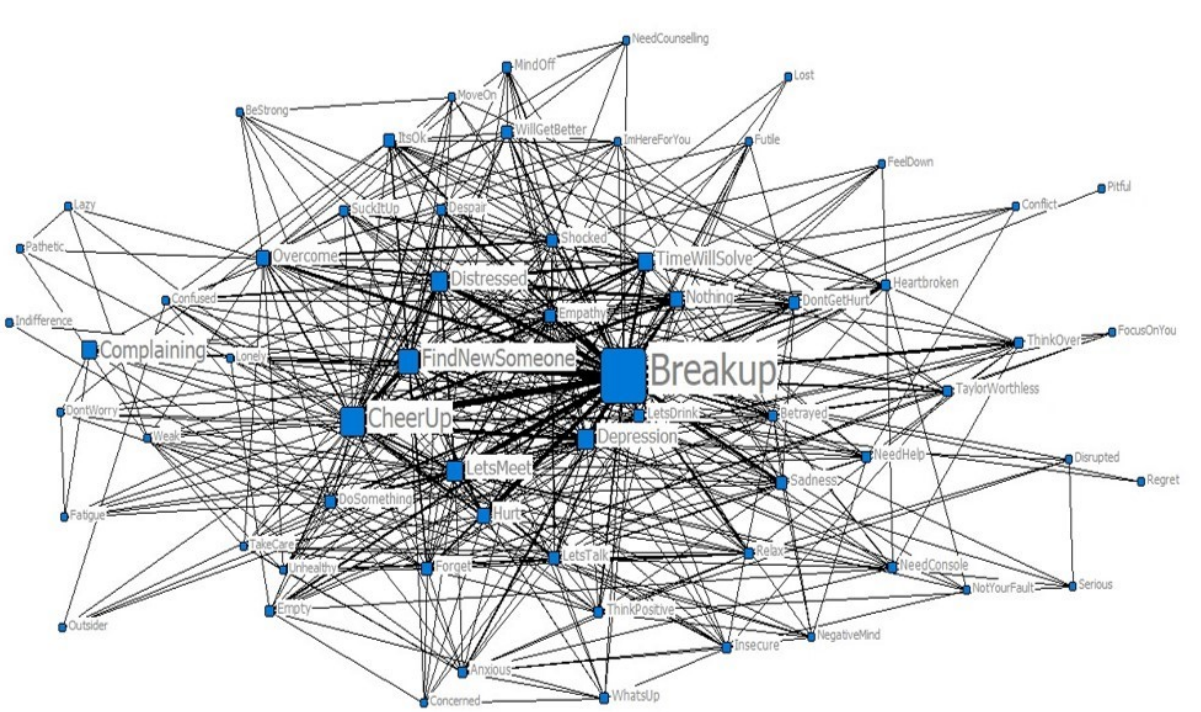

Subclinical distress

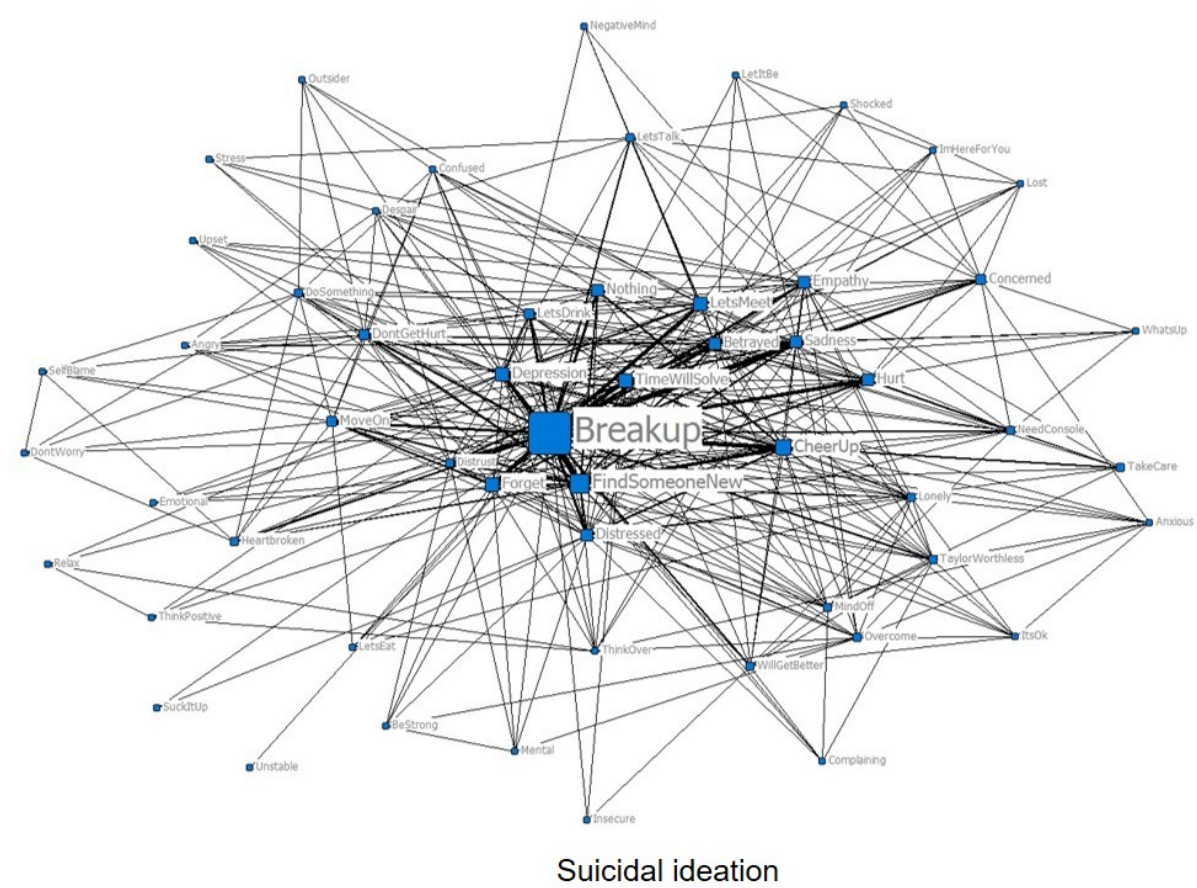

Suicidal ideation

Figure 7. Semantic network of the responses of Korean participants 


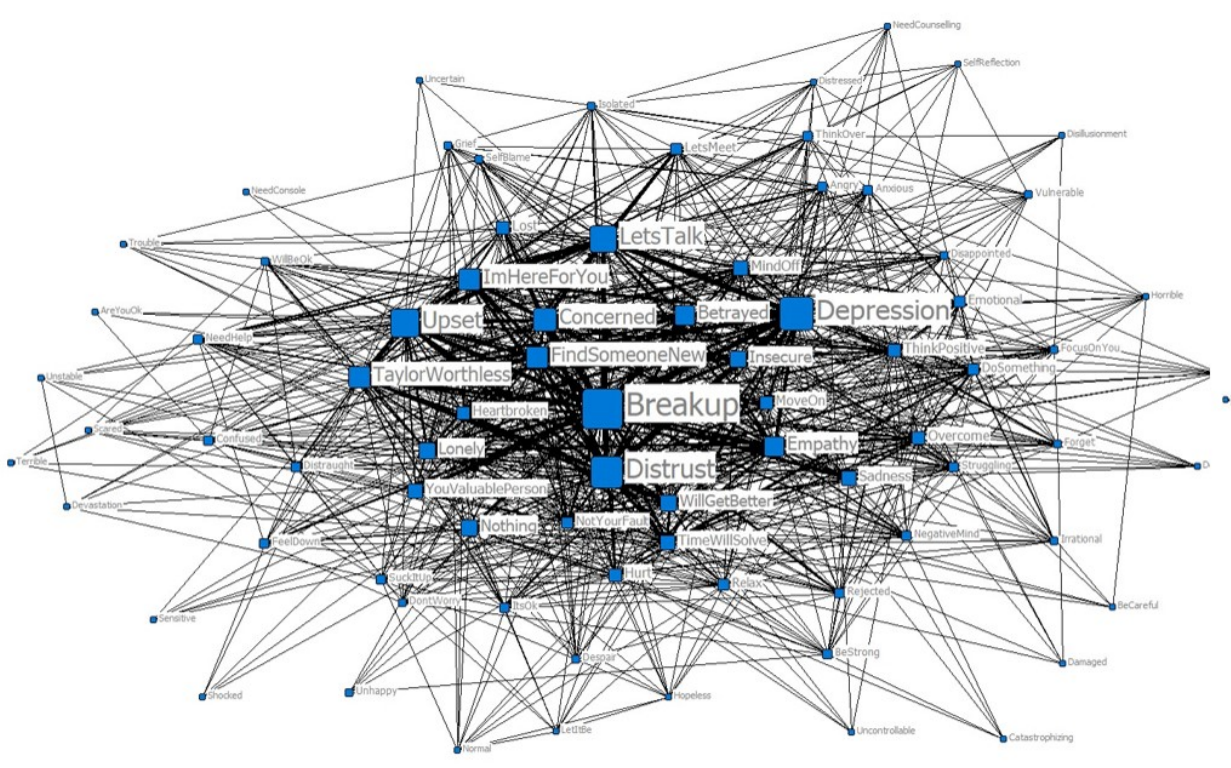

Subclinical distress

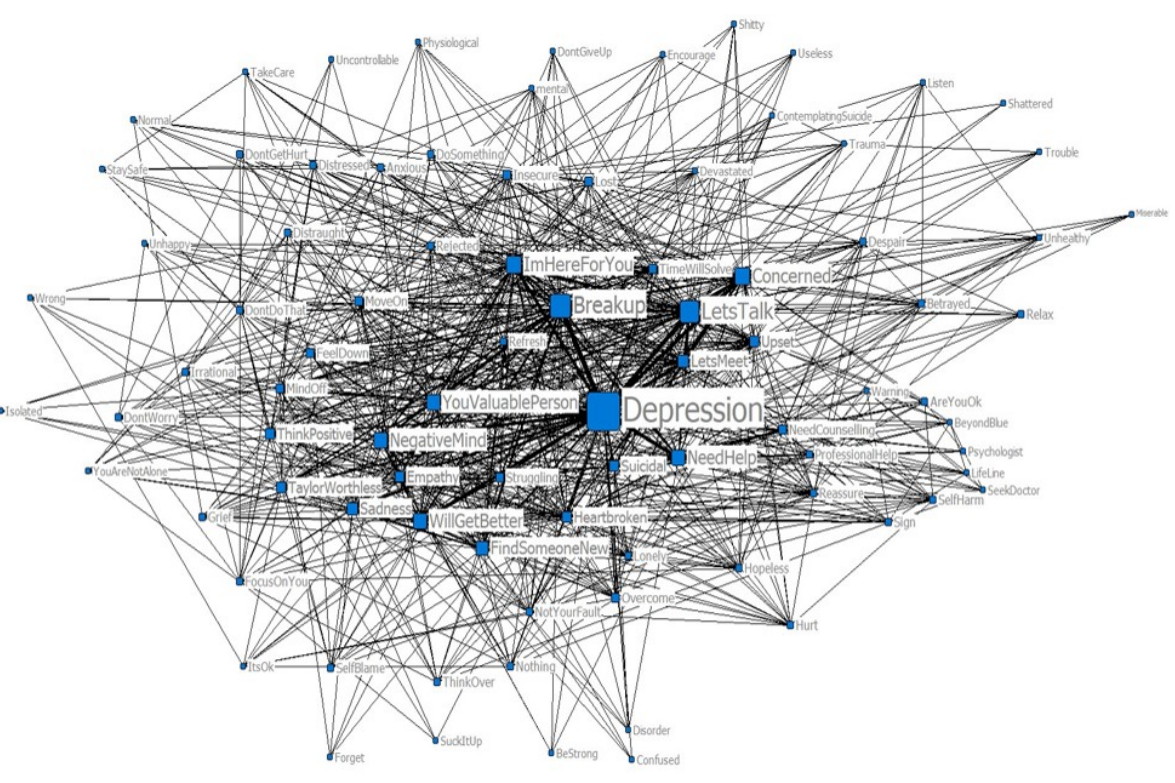

Suicidal ideation

Figure 8. Semantic network of the responses of Australian participants 


\section{References}

An, S. \& Lee, H. (2017a). An exploratory study for developing a social stigma scale toward suicidal people, Health and Social Welfare Review, 37(2), 325-357.

An, S. \& Lee, H. (2017b). Perception and attitudes toward suicide: Acceptance of negative emotions and suicide literacy, Korean Journal of Social Welfare Research, 52, 95117.

American Psychiatric Association (2000). Diagnostic and statistical manual of mental disorders fourth (DSM-IV-TR). Washington, DC: American Psychiatric Association.

Bastian, B., Kuppens, P., Hornsey, M. J., Park, J., Koval, P., \& Uchida, Y. (2012). Feeling bad about being sad: The role of social expectancies in amplifying negative mood. Emotion, 12(1), 69.

Borgatti, S. P., Everett, M. G., \& Johnson, J. C. (2013). Analyzing social networks. London: Sage

Butler, E. A., Lee, T. L., \& Gross, J. J. (2007). Emotion regulation and culture: Are the social consequences of emotion suppression culture-specific?. Emotion, 7(1), 30-48.

Chang, M. X.-L., Jetten, J., Cruwys, T., \& Haslam, C. (2017). Cultural identity and the expression of depression: A social identity perspective. Journal of Community \& Applied Social Psychology, 27(1), 16-34. https://doi.org/10.1002/casp.2291

Chou, W. Y. S., Hunt, Y. M., Beckjord, E. B., Moser, R. P., \& Hesse, B. W. (2009). Social media use in the United States: implications for health communication. Journal of Medical Internet Research, 11(4), e48. Retrieved from https://www.ncbi.nlm.nih.gov/pmc/articles/PMC2802563/

Chu, J. P., Hsieh, K. Y., \& Tokars, D. A. (2011). Help-seeking tendencies in Asian Americans with suicidal ideation and attempts. Asian American Journal of Psychology, 2(1), 2538.

Colucci, E. (2013). Culture, cultural meaning(s), and suicide. In E. Colucci \& D. Lester (Eds.), Suicide and culture: Understanding the context, pp. 25-46. Cambridge, MA: Hogrefe Publishing.

Colucci, E. (2013). Cultural meaning(s) of suicide: A cross-cultural study. In E. Colucci \& D. Lester (Eds.), Suicide and culture: Understanding the context, pp. 94-195. Cambridge, MA: Hogrefe Publishing.

Colucci, E., Kelly, C. M., Minas, H., Jorm, A. F., \& Chatterjee, S. (2010). Mental Health First Aid guidelines for helping a suicidal person: a Delphi consensus study in India. International Journal of mental health Systems, 4(1), 4.

Colucci, E., Kelly, C. M., Minas, H., Jorm, A. F., \& Nadera, D. (2010). Mental Health First Aid guidelines for helping a suicidal person: a Delphi consensus study in the Philippines. International journal of mental health systems, 4(1), 32. 
Colucci, E., Kelly, C. M., Minas, H., Jorm, A. F., \& Suzuki, Y. (2011). Mental health first aid guidelines for helping a suicidal person: a Delphi consensus study in Japan. International journal of mental health systems, 5(1), 12.

Colucci, E. \& Lester, D. (2013). Suicide and culture: Understanding the context. Cambridge, MA: Hogrefe Publishing.

Cowgell, V. G. (1977). Interpersonal effects of a suicidal communication. Journal of Consulting and Clinical Psychology, 45(4), 592-599.

Cruwys, T., An, S., Chang, M. X. L., \& Lee, H. (2018). Suicide literacy predicts the provision of more appropriate support to people experiencing psychological distress. Psychiatry Rresearch, 264, 96-103.

De Vaus, J., Hornsey, M. J., Kuppens, P., \& Bastian, B. (2018). Exploring the East-West divide in prevalence of affective disorder: A case for cultural differences in coping with negative emotion. Personality and Social Psychology Review, 22(3), 285-304.

Diener, E., \& Diener, C. (1996). Most people are happy. Psychological science, 7(3), 181185.

Dolev-Cohen, M., \& Barak, A. (2013). Adolescents' use of Instant Messaging as a means of emotional relief. Computers in Human Behavior, 29(1), 58-63.

Domino, G. (2005). Cross-cultural attitudes towards suicide: the SOQ and a personal odyssey. Archives of Suicide Research, 9(2), 107-122.

Drieger, P. (2013). Semantic network analysis as a method for visual text analytics. ProcediaSocial and Behavioral Sciences, 79, 4-17.

Eagles, J. M., Carson, D. P., Begg, A., \& Naji, S. A. (2003). Suicide prevention: A study of patients' views. British Journal of Psychiatry, 182, 261-265.

Eskin, M., Voracek, M., Stieger, S., \& Altinyazar, V. (2011). A cross-cultural investigation of suicidal behavior and attitudes in Austrian and Turkish medical students. Social Psychiatry and Psychiatric Epidemiology, 46(9), 813-823.

Freedenthal, S., \& Stiffman, A. R. (2007). “They might think I was crazy": young American Indians' reasons for not seeking help when suicidal. Journal of Adolescent Research, 22(1), 58-77.

Freeman, L. C. (2000). Visualizing social networks. Journal of Social Structure, 1(1), 1-15. Retrieved from https://www.cmu.edu/joss/content/articles/volume1/Freeman.html

Goldston, D. B., Molock, S. D., Whitbeck, L. B., Murakami, J. L., Zayas, L. H., \& Hall, G. C. N. (2008). Cultural considerations in adolescent suicide prevention and psychosocial treatment. American Psychologist, 63(1), 14-31.

Hall, J. A. (2018). When is social media use social interaction? Defining mediated social interaction. New Media \& Society, 20(1), 162-179. 
Han, J., Batterham, P. J., Calear, A. L., \& Ma, J. (2018). Seeking professional help for suicidal ideation: a comparison between Chinese and Australian university students. Psychiatry Research, 270, 807-814.

Hawton, K., Houston, K., \& Shepperd, R. (1999). Suicide in young people: Study of 174 cases, aged under 25 years, based on coroners' and medical records. The British Journal of Psychiatry, 175, 271-276.

Heppner, P. P., Heppner, M. J., Lee, D. G., Wang, Y. W., Park, H. J., \& Wang, L. F. (2006). Development and validation of a collectivist coping styles inventory. Journal of Counseling Psychology, 53(1), 107-125.

Hjelmeland, H. (2010). Cultural research in suicidology: Challenges and opportunities. Suicidology Online, 1(1), 34-52.

Hjelmeland, H. (2011). Cultural Context Is Crucial in Suicide Research and Prevention. Crisis, 32(2), 61-64.

Hjelmeland, H., \& Knizek, B. L. (2011). Methodology in suicidological research-contribution to the debate. Suicidology Online, 2, 8-10.

Holmes, E. P., Corrigan, P. W., Williams, P., Canar, J., \& Kubiak, M. A. (1999). Changing attitudes about schizophrenia. Schizophrenia Bulletin, 25(3), 447-456.

Hufford, M. R. (2001). Alcohol and suicidal behavior. Clinical Psychology Review, 21(5), 797-811.

Isometsä, E. T. (2001). Psychological autopsy studies-a review. European Psychiatry, 16(7), 379-385.

Jorm, A. F., Griffiths, K. M., Christensen, H., Korten, A. E., Parslow, R. A., \& Rodgers, B. (2003). Providing information about the effectiveness of treatment options to depressed people in the community: a randomized controlled trial of effects on mental health literacy, help-seeking and symptoms. Psychological Medicine, 33(6), 10711079.

Jorm, A. F., Ross, A. M., \& Colucci, E. (2018). Cross-cultural generalizability of suicide first aid actions: an analysis of agreement across expert consensus studies from a range of countries and cultures. BMC Psychiatry, 18(1), 58.

Kroenke, K., Spitzer, R. L., \& Williams, J. B. (2001). The PHQ-9: validity of a brief depression severity measure. Journal of General Internal Medicine, 16(9), 606-613.

Lester, D., \& Walker, R. L. (2006). The stigma for attempting suicide and the loss to suicide prevention efforts. Crisis: The Journal of Crisis Intervention and Suicide Prevention, 27(3), 147-148.

Leydesdorff, L., \& Welbers, K. (2011). The semantic mapping of words and co-words in contexts. Journal of Informetrics, 5(3), 469-475. doi.org/10.1016/j.joi.2011.01.008

Linda, W. P., Marroquín, B., \& Miranda, R. (2012). Active and passive problem solving as 
moderators of the relation between negative life event stress and suicidal ideation among suicide attempters and non-attempters. Archives of Suicide Research, 16(3), 183-197. https://doi.org/10.1080/13811118.2012.695233

Markus, H. R., \& Kitayama, S. (1991). Culture and the self: Implications for cognition, emotion, and motivation. Psychological Review, 98(2), 224-253.

Matsubayashi, T., \& Ueda, M. (2011). The effect of national suicide prevention programs on suicide rates in 21 OECD nations. Social Science and Medicine, 73(9), 1395-1400. https://doi.org/10.1016/j.socscimed.2011.08.022

Matsumoto, D., Yoo, S. H., \& Nakagawa, S. (2008). Culture, emotion regulation, and adjustment. Journal of Personality and Social Psychology, 94(6), 925-937.

Morrison, L. L., \& Downey, D. L. (2000). Racial differences in self-disclosure of suicidal ideation and reasons for living: Implications for training. Cultural Diversity and Ethnic Minority Psychology, 6(4), 374-386.

Nock, M. K., Borges, G., Bromet, E. J., Cha, C. B., Kessler, R. C., \& Lee, S. (2008). Suicide and suicidal behavior. Epidemiologic Reviews, 30(1), 133-154.

OECD (2018), Suicide rates (indicator). doi: 10.1787/a82f3459-en (Accessed on 14 November 2018)

Owens, C., Owen, G., Belam, J., Lloyd, K., Rapport, F., Donovan, J., \& Lambert, H. (2011). Recognising and responding to suicidal crisis within family and social networks: qualitative study. British Medical Journal, 343, d5801. Retrieved from https://www.bmj.com/content/343/bmj.d5801.short

Quan-Haase, A., \& Young, A. L. (2010). Uses and gratifications of social media: A comparison of Facebook and instant messaging. Bulletin of Science, Technology \& Society, 30(5), 350-361. doi.org/10.1177/0270467610380009

Rudestam, K. E. (1971). Stockholm and Los Angeles: A cross-cultural study of the communication of suicidal intent. Journal of Consulting and clinical Psychology, $36(1), 82-90$.

Taylor-Rodgers, E., \& Batterham, P. J. (2014). Evaluation of an online psychoeducation intervention to promote mental health help seeking attitudes and intentions among young adults: randomised controlled trial. Journal of Affective Disorders, 168, 65-71.

Tzeng, W. C., \& Lipson, J. G. (2004). The cultural context of suicide stigma in Taiwan. Qualitative Health Research, 14(3), 345-358.

Uchida, Y., \& Kitayama, S. (2009). Happiness and unhappiness in east and west: Themes and variations. Emotion, 9(4), 441-456.

Valkenburg, P. M., Peter, J., \& Schouten, A. P. (2006). Friend networking sites and their relationship to adolescents' well-being and social self-esteem. CyberPsychology \& Behavior, 9(5), 584-590. 
Van Atteveldt, W. V. (2008). Semantic network analysis: Techniques for extracting, representing and querying media content. Charleston, SC: BookSurge Publishers. Retrieved from http://dare.ubvu.vu.nl/bitstream/handle/1871/15964/complete\%20dissertation.pdf?seq $\underline{\text { uence }=5}$

Walker, R. L., Lester, D., \& Joe, S. (2006). Lay theories of suicide: An examination of culturally relevant suicide beliefs and attributions among African Americans and European Americans. Journal of Black Psychology, 32(3), 320-334.

World Health Organization (2010). Toward evidence-based suicide prevention programmes. Retrieved from http://apps.who.int/iris/bitstream/handle/10665/207049/9789290614623_eng.pdf?sequen $\mathrm{ce}=1$ \& isAllowed $=\mathrm{y}$

World Health Organization (2017). World Health Statistics 2017: Monitoring health for the SDGs. World Health Organization Publishing. 